\title{
CONHECIMENTO, ATITUDES E PRÁTICAS DOS PROFISSIONAIS DE SAÚDE DA ATENÇÃO PRIMÁRIA SOBRE PREVENÇÃO DO CÂNCER DE MAMA
}

\author{
KNOWLEDGE, ATTITUDES AND PRACTICES OF PRIMARY CARE HEALTH \\ PROFESSIONALS ABOUT BREAST CANCER PREVENTION
}

\section{Rafaela Cristina Araújo Gomes ${ }^{1}$ * Adriana Gomes Nogueira Ferreira ${ }^{2}$ Glaucya Santos Madeira ${ }^{3}$ * Aldo Lopes da Costa Júnior ${ }^{4 *}$ * Patrícia Neyva da Costa Pinheiro ${ }^{5}$ * Vivian Saraiva Veras ${ }^{6}$}

\begin{abstract}
RESUMO
Objetivo: Identificar o conhecimento, atitudes e práticas de profissionais de saúde da atenção primária sobre prevenção do câncer de mama. Métodos: Trata-se de uma revisão integrativa da literatura realizada em dezembro de 2020. As bases de dados selecionadas para realizar as buscas foram: Biblioteca Virtual de Saúde (BVS - BIREME), Scopus (Elsevier), Web of Science, Cumulative Index to Nursing and Allied Health Literature (CINAHL - EBSCO) e Pubmed (NLM - NCBI). Resultados: Foram incluídos e analisados 11 artigos. O conhecimento foi analisado por sete estudos, demonstrando-se adequado em cinco, de acordo com as respostas da maioria dos profissionais de saúde. Contudo, dos oito estudos que analisaram as atitudes, em apenas dois a maioria dos profissionais apresentaram-nas de forma correta e dos seis artigos que verificaram as práticas, somente em um, os profissionais realizavam-nas corretamente. Conclusão: O conhecimento analisado na maioria dos estudos foi considerado adequado, porém isso não foi refletido nas atitudes e práticas. Sendo assim, é importante reforçar a relação das atitudes e práticas com o conhecimento adquirido, os quais se tornam um tripé para a eficácia da abordagem junto à mulher para a prevenção do câncer de mama.
\end{abstract}

Palavras-chave: Neoplasias da mama; Conhecimentos, Atitudes e Prática em Saúde; Atenção Primária à Saúde; Pessoal de Saúde.

\begin{abstract}
Objectives: To identify the knowledge, attitudes and practices of primary care health professionals about breast cancer prevention. Methods: This is an integrative literature review carried out in December 2020. The databases selected to perform the searches were: Virtual Health Library (BVS - BIREME), Scopus (Elsevier), Web of Science, Cumulative Index to Nursing and Allied Health Literature (CINAHL - EBSCO) and Pubmed (NLM - NCBI). Results: 11 articles were included and analyzed. Knowledge was analyzed by seven studies, proving to be adequate in five, according to the responses of most health professionals. However, of the eight studies that analyzed attitudes, in only two did the majority of professionals present them correctly, and of the six articles that verified the practices, only in one did the professionals perform them correctly. Conclusion: The knowledge analyzed in most studies was considered adequate, but this was not reflected in attitudes and practices. Therefore, it is important to reinforce the relationship of attitudes and practices with acquired knowledge, which become a tripod for the effectiveness of the approach with women for the prevention of breast cancer.
\end{abstract}

Keywords: Breast Neoplasms; Health Knowledge, Attitudes, Practice; Primary Health Care; Health Personnel.

\footnotetext{
${ }^{1}$ Universidade Federal do Maranhão, Imperatriz, Brasil. ORCID: 0000-0002-4607-4756

${ }^{2}$ Universidade Federal do Maranhão, Imperatriz, Brasil. ORCID: 0000-0002-7107-1151

${ }^{3}$ Universidade Federal do Maranhão, Imperatriz, Brasil. ORCID: 0000-0002-6577-8839

${ }^{4}$ Universidade Federal do Maranhão, Imperatriz, Brasil. ORCID: 0000-0002-4216-4690

${ }^{5}$ Universidade Federal do Ceará, Fortaleza, Brasil. ORCID: 0000-0001-7022-8391

${ }^{6}$ Universidade da Integração Internacional da Lusofonia Afro-Brasileira, Ceará, Brasil. ORCID: 0000-0003-3267-3712
} 


\section{INTRODUÇÃO}

As ações de prevenção e promoção da saúde na atenção primária, envolvem uma equipe multiprofissional de médicos, enfermeiros, dentistas, técnicos e demais profissionais de saúde, sendo que o Conhecimento (dimensão cognitiva, recordação de fatos e habilidade para aplicar o conhecimento na resolução dos problemas), a Atitude (percepção de adoção de medidas preventivas adequadas em saúde e sua importância) e a Prática (comportamento pessoal do indivíduo e aquisição de habilidades) (CAP) dos mesmos, relacionadas à detecção precoce do câncer de mama (CM), se tornam essenciais para a integralidade dos serviços prestados à mulher, apresentando um impacto positivo para redução da morbimortalidade ${ }^{(1-3)}$.

O câncer de mama é a neoplasia maligna mais comum em mulheres, no qual a sobrevida dessas pacientes diagnosticadas em países desenvolvidos é de $85 \%$, já em países em desenvolvimento esse número chega a $60 \%$ (4). Caracterizada de origem multifatorial, o desenvolvimento da doença pode envolver fatores biológicos e endócrinos relacionados à vida reprodutiva, ao comportamento e ao estilo de vida. Vale ressaltar que graças ao processo de transição sociodemográfica de doenças crônicodegenerativas, o câncer está intensificando-se na sociedade devido às mudanças no estilo de vida, hábitos alimentares, envelhecimento, exposição a agentes cancerígenos, obesidade, stress, entre outros ${ }^{(5-7)}$.

Para proporcionar maior sobrevida às mulheres, reduzir o surgimento de complicações, e até mesmo reduzir os fatores de risco para a doença, é fundamental que o profissional de saúde desempenhe o papel de alertar e orientar a população feminina sobre a importância da prevenção de tumores mamários ${ }^{(8-9)}$. O fato de os profissionais apresentarem insuficiência no conhecimento sobre as formas e meios de prevenção da doença, é uma tarefa que aparece como um do serviço público de saúde e pode ocasionar adversos problemas, principalmente a não detecção da doença de forma precoce.

$\mathrm{Na}$ atenção primária a saúde é onde ocorrem as principais ações de promoção da saúde, predominantemente as preventivas. Apesar disso, ainda é necessário buscar fortalecimento das estratégias que ocorrem para o conhecimento, atitude e pratica, principalmente as relacionadas a capacitação das equipes de saúde da família, contribuindo para que os profissionais envolvidos possam reconhecer e realizarem seus papeis como educadores em saúde ${ }^{(10)}$.

A falta de conhecimento desses profissionais pode afetar as práticas e atitudes relacionadas a prevenção do câncer de mama. Atualmente, há uma escassez de informações sobre os níveis de CAP dos profissionais, o que é essencial para o desenvolvimento de estratégias abrangente de prevenção e controle de câncer mamário ${ }^{(11)}$. Os mesmos https://doi.org/10.31011/reaid-2021-v.95-n.35-art.1198 Rev Enferm Atual In Derme v. 95, n. 35, 2021 e-021123 
são agentes de mudança, oferecem aconselhamento útil para promoção da saúde, e quando os mesmos possuem o conhecimento, passam a ter cerca de três vezes mais probabilidade de realizar as orientações recomendadas ${ }^{(12)}$.

Em relação a atitude, esta é conhecida como habilidades não técnicas, essências para o fornecimento de informações, como se sentir preparado e capaz de estabelecer um vínculo e explorar os problemas e eventuais risco para o paciente ${ }^{(13)}$. Nessa vertente, os três elementos CAP dos profissionais de saúde devem estar alinhados, a fim de proporcionar condições para a promoção da autonomia, independência das pacientes e um processo de cuidar baseado na propagação da prevenção do CM.

Os profissionais de saúde devem estar em constante atualização sobre os temas relacionados à doença e sua prevenção, buscando melhorar seu conhecimento, suas atitudes e práticas, assim, fornecendo as orientações necessárias e identificando o melhor meio de promover o cuidado baseado na necessidade de cada paciente ${ }^{(14)}$. Baseado nessa abordagem, o presente artigo tem como objetivo, por meio de uma revisão integrativa da literatura, identificar o conhecimento, atitude e práticas de profissionais de saúde da atenção primária sobre prevenção do câncer de mama.

\section{MÉTODOS}

Trata-se de uma revisão integrativa da literatura que tem por objetivo apresentar uma ampla visão sobre o assunto pesquisado ${ }^{(15)}$. Assim, foram seguidos os seis passos para elaboração desta revisão, quais sejam: formulação da questão norteadora, seleção dos descritores para busca, seleção das bases de dados, extração dos estudos selecionados das bases, análise e síntese dos achados e por fim, a apresentação deles.

Para auxiliar a formulação da questão norteadora da presente pesquisa, utilizou-se a estratégia mnemônica PICo, metodologia voltada para a pesquisa não-clínica, no qual o acrônimo P representa problema, paciente ou população, sendo neste caso utilizado os profissionais de saúde; o I relacionado ao interesse da pesquisa, indicado como o conhecimento, atitudes e práticas sobre prevenção do câncer de mama; e o Co informando o contexto, que é a atenção primária à saúde ${ }^{(16)}$. Dessa forma, a questão norteadora elaborada para este estudo foi: Qual o conhecimento, atitudes e práticas dos profissionais de saúde da atenção primária sobre prevenção do câncer de mama?

A seleção dos descritores para realizar as buscas foi definida através dos descritores em ciências da saúde (DeCS) e Medical Subject Headings (MeSH), onde cada acrônimo do PICo auxiliou na identificação de termos que se adequavam na pesquisa, 
organizados da forma apresentada no quadro

a seguir:

Quadro 1 - Descritores utilizados para realizar as buscas da presente pesquisa.

\begin{tabular}{|c|c|c|}
\hline PICo & DeCS (português) & MeSH (inglês) \\
\hline $\begin{array}{l}\text { P- Profissionais } \\
\text { de saúde }\end{array}$ & $\begin{array}{c}\text { pessoal de saúde } \\
\text { psicologia } \\
\text { odontólogos } \\
\text { nutricionistas } \\
\text { assistentes sociais } \\
\text { terapia ocupacional } \\
\text { médicos } \\
\text { enfermeiras e enfermeiros } \\
\text { técnicos de enfermagem } \\
\text { agentes comunitários de saúde } \\
\text { educação física e treinamento }\end{array}$ & $\begin{array}{c}\text { health personnel } \\
\text { psychology } \\
\text { dentists } \\
\text { nutritionists } \\
\text { social workers } \\
\text { occupational therapy } \\
\text { physicians } \\
\text { nurses } \\
\text { licensed practical nurses } \\
\text { community health workers } \\
\text { physical education and training }\end{array}$ \\
\hline $\begin{array}{c}\text { I- Conhecimento } \\
\text { sobre prevenção } \\
\text { do câncer de } \\
\text { mama }\end{array}$ & $\begin{array}{c}\text { conhecimentos, atitudes e prática em saúde } \\
\text { atitude do pessoal de saúde } \\
\text { prevenção primária } \\
\text { prevenção e controle } \\
\text { prevenção secundária } \\
\text { neoplasias da mama }\end{array}$ & $\begin{array}{l}\text { health knowledge, attitudes, practice } \\
\text { attitude of health personnel } \\
\text { primary prevention } \\
\text { prevention and control } \\
\text { secondary prevention } \\
\text { breast neoplasms }\end{array}$ \\
\hline $\begin{array}{c}\text { Co- Atenção } \\
\text { primária à saúde }\end{array}$ & atenção primária à saúde & primary health care \\
\hline
\end{tabular}

Fonte: Elaborado pelos autores.

Além disso, foi realizada uma chave de busca única utilizando os descritores selecionados e os operadores booleanos AND e OR à medida que foi necessário. Cabe ressaltar que se optou por utilizar os termos em inglês devido a maioria das bases serem internacionais e este idioma ser obrigatório nos títulos e resumos em publicações. Sendo assim, a chave de busca formada e utilizada em todas as bases foi a seguinte: ((health personnel OR psychology OR dentists OR nutritionists OR social workers OR occupational therapy $O R$ physicians $O R$ nurses OR licensed practical nurses OR community health workers $O R$ physical education and training) AND (health knowledge, attitudes, practice OR attitude of health personnel) AND (primary prevention OR prevention and control OR secondary prevention) AND (breast neoplasms) AND (primary health care)).

As bases de dados selecionadas para realizar as buscas foram a Biblioteca Virtual de Saúde (BVS - BIREME), Scopus (Elsevier), Web of Science (Coleção Principal

- Clarivate Analytics), Cumulative Index to Nursing and Allied Health Literature (CINAHL - EBSCO) e Pubmed (NLM NCBI). Cabe ressaltar que nas bases BVS, CINAHL e Pubmed a pesquisa foi realizada na opção de busca básica, onde ainda na BVS foi utilizado como item de busca a opção 
título, resumo e assunto, já nas bases Scopus e

Web of Science, foi como busca avançada, e houve uma exceção na chave de busca para Web of Science pois foi necessário acrescentar antes o termo "TS=", que se refere a tópico (título, resumo e palavras-chave).

Foram incluídos artigos disponíveis na íntegra, com acesso livre, nos idiomas português, inglês e espanhol, que tivessem como público-alvo os profissionais de saúde e que abordassem os conhecimentos, atitudes e/ou prática dos profissionais sobre o câncer de mama e sua prevenção. Assim, foram excluídos artigos duplicados e estudos sobre outras doenças.

Dessa forma, a busca foi realizada em dezembro de 2020 por dois pesquisadores paralelamente nas etapas de identificação, seleção e extração dos dados de forma independente, de modo que houvesse uma segunda opinião sobre a inclusão ou exclusão dos estudos encontrados. Para padronizar a coleta, a busca pelas bases foi realizada no portal de periódicos da Coordenação de Aperfeiçoamento de Pessoal de Nível Superior (CAPES), por meio da Comunidade Acadêmica Federada (CAFe), selecionando a instituição de ensino superior Universidade Federal do Maranhão (UFMA).

\section{RESULTADOS}

Foram encontrados 230 estudos no total nas cinco bases selecionadas, os estágios de inclusão dos estudos até a seleção final estão descritos no fluxograma abaixo, adaptado do PRISMA ${ }^{(17)}$. 
Figura 1 - Fluxograma com estratégia de busca para seleção dos artigos.

$\mathrm{N}^{\mathrm{o}}$ de estudos identificados nas bases de dados:

$(\mathrm{n}=230)$

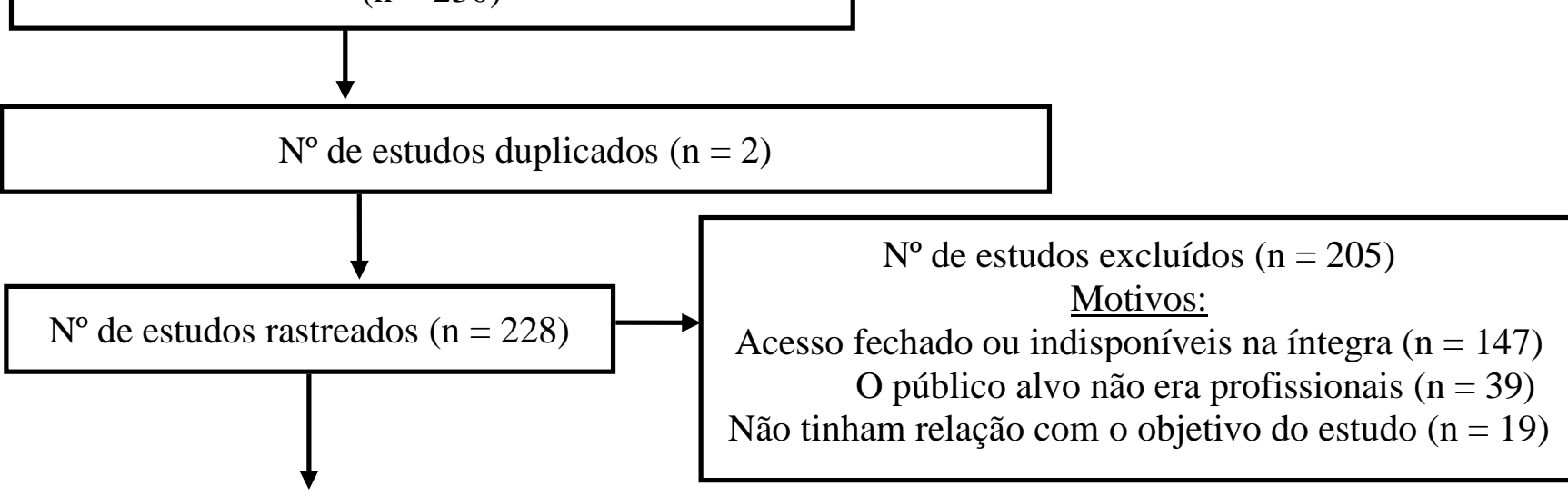

$\mathrm{N}^{\mathrm{o}}$ de artigos em texto completo avaliados para elegibilidade $(n=23)$

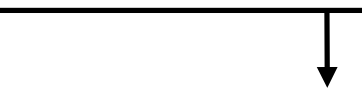

$\mathrm{N}^{\mathrm{o}}$ de artigos incluídos $(\mathrm{n}=11)$
$\mathrm{N}^{\circ}$ de artigos completos excluídos por não responderem à questão de pesquisa $(n=12)$

Fonte: Adaptado do PRISMA ${ }^{(17)}$.

Foram excluídos dois artigos por serem duplicados e assim foi realizada a leitura dos títulos e resumos dos 228 artigos selecionados, sendo excluídos 205 destes, por serem de acesso fechado e estarem indisponíveis na íntegra, com público-alvo que não fossem profissionais e por não responderem à questão de pesquisa. Dessa forma, 23 artigos foram eleitos e acessados para leitura na íntegra, assim, foram extraídos das bases de dados por meio de download em Portable Document Format (PDF) e foram analisados por dois pesquisadores.
Posteriormente a leitura e análise dos artigos, os pesquisadores iniciaram uma discussão sobre a seleção final dos estudos, entrando em um consenso sobre a inclusão de 11 artigos para síntese qualitativa, que consistiu em reunir os principais dados dos estudos e a resposta contida neles à questão norteadora desta revisão. Neste sentido, a apresentação da síntese e dos achados foi organizada em dois quadros, o primeiro contendo uma caracterização dos artigos com autores, ano de publicação, periódico publicado, local de realização do estudo e 
idioma, e o segundo contendo as resultados e conclusões.

especificidades com título, objetivo, método,

Quadro 2 - Caracterização das publicações incluídas na revisão integrativa.

\begin{tabular}{|c|c|c|c|c|}
\hline ID & Autores & Ano & Periódico & Local de estudo \\
\hline A1 ${ }^{(18)}$ & $\begin{array}{l}\text { Taylor et } \\
\text { al. }\end{array}$ & 1994 & Public Health Reports & Washington (EUA) \\
\hline $\mathrm{A} 2^{(19)}$ & $\begin{array}{l}\text { Escher e } \\
\text { Sappino }\end{array}$ & 2000 & $\begin{array}{l}\text { Kluwer Academic } \\
\text { Publishers }\end{array}$ & Genebra (Suíça) \\
\hline A $3^{(20)}$ & $\begin{array}{l}\text { Benard et } \\
\text { al. }\end{array}$ & 2011 & $\begin{array}{c}\text { Journal of Women's } \\
\text { Health }\end{array}$ & EUA \\
\hline $\mathrm{A} 4^{(21)}$ & $\begin{array}{l}\text { Collins et } \\
\text { al. }\end{array}$ & 2014 & The Breast & Melbourne (Austrália) \\
\hline $\mathrm{A} 5^{(22)}$ & Haas et al. & 2015 & $\begin{array}{l}\text { Journal of General } \\
\text { Internal Medicine }\end{array}$ & $\begin{array}{c}\text { Boston, Líbano, Filadélfia e } \\
\text { Vermont (EUA) }\end{array}$ \\
\hline A6 ${ }^{(23)}$ & $\begin{array}{l}\text { Erdem e } \\
\text { Toktas }\end{array}$ & 2016 & $\begin{array}{l}\text { BioMed Research } \\
\text { International }\end{array}$ & Diyarbakır (Turquia) \\
\hline A7 ${ }^{(24)}$ & $\begin{array}{l}\text { Nazzal et } \\
\text { al. }\end{array}$ & 2016 & $\begin{array}{c}\text { Asian Pacific Journal of } \\
\text { Cancer Prevention }\end{array}$ & Cisjordânia \\
\hline A ${ }^{(25)}$ & Haas et al. & 2017 & $\begin{array}{l}\text { Journal of General } \\
\text { Internal Medicine }\end{array}$ & $\begin{array}{c}\text { Boston, Líbano, Filadélfia e } \\
\text { Vermont (EUA) }\end{array}$ \\
\hline A9 ${ }^{(26)}$ & Smith et al. & 2017 & $\begin{array}{l}\text { British Journal of General } \\
\text { Practice }\end{array}$ & $\begin{array}{l}\text { Inglaterra, Irlanda do Norte e } \\
\text { País de Gales (Reino Unido) }\end{array}$ \\
\hline $\begin{array}{c}\mathrm{A} 10 \\
(27)\end{array}$ & $\begin{array}{l}\text { Hallowell } \\
\text { et al. }\end{array}$ & 2018 & Preventive Medicine & Brasil \\
\hline $\begin{array}{c}\mathrm{A} 11 \\
(28)\end{array}$ & $\begin{array}{c}\text { Schoenborn } \\
\text { et al. }\end{array}$ & 2020 & $\begin{array}{l}\text { The American Geriatrics } \\
\text { Society }\end{array}$ & Maryland (EUA) \\
\hline
\end{tabular}

Fonte: Elaborado pelos autores. 
Quadro 3 - Distribuição das publicações incluídas na revisão integrativa segundo objetivo, métodos, e conhecimentos, atitudes e práticas.

\begin{tabular}{|c|c|c|c|}
\hline ID & Objetivo & Método & Conhecimento, Atitudes e Práticas \\
\hline A1 ${ }^{(18)}$ & $\begin{array}{l}\text { Descrever a análise, } \\
\text { mobilização e envolvimento de } \\
\text { comunidades médicas } \\
\text { em dois municípios direcionados à } \\
\text { intervenção na comunidade de } \\
\text { Hutchinson, centro de Washington, no } \\
\text { Projeto de Rastreio do Câncer de mama. }\end{array}$ & $\begin{array}{l}\text { Tipo de estudo: Estudo demográfico } \\
\text { e estudo descritivo qualitativo; } \\
\text { Local de estudo: } \\
\text { Washington (EUA); } \\
\text { Participantes: } 158 \text { médicos (98 do } \\
\text { Norte e } 60 \text { do Sul) entre eles: } \\
\text { Médicos da família, Médicos } \\
\text { internistas gerais, Ginecologistas, } \\
\text { Oncologistas, Oncologista de } \\
\text { radiação, Radiologistas, Cirurgiões. }\end{array}$ & $\begin{array}{l}\text { Conhecimento: A maioria dos médicos acreditavam que } \\
\text { a triagem reduz a mortalidade, detecta câncer em estágio } \\
\text { inicial e que não se detecta câncer só com o exame } \\
\text { físico das mamas. Além disso apontaram o alto custo da } \\
\text { mamografia e o seguro inadequado como fatores } \\
\text { negativos para a adesão das pacientes. } \\
\text { Atitude: Em relação as percepções, a maioria dos } \\
\text { médicos da comunidade norte e uma minoria do sul } \\
\text { disseram existir um consenso sobre o intervalo da } \\
\text { mamografia. Foi discutido ser essencial que a equipe de } \\
\text { funcionários das clínicas tenha um mínimo de } \\
\text { conhecimento sobre o câncer de mama e os } \\
\text { procedimentos de triagem, visto que são quem tem o } \\
\text { primeiro contato com os pacientes e podendo promover } \\
\text { uma diminuição das barreiras para triagem. } \\
\text { Práticas: Foi verificado que a maioria dos médicos de } \\
\text { ambas as comunidades realizavam mamografia } \\
\text { anualmente. }\end{array}$ \\
\hline $\mathrm{A} 2^{(19)}$ & $\begin{array}{l}\text { Avaliar o conhecimento, atitude } \\
\text { percepção dos médicos sobre seu papel } \\
\text { nos testes genéticos } \\
\text { para predisposição ao câncer de mama } \\
\text { ovariano. }\end{array}$ & $\begin{array}{l}\text { Tipo de estudo: Transversal, } \\
\text { descritivo, quali-quanti; } \\
\text { Local de estudo: } \\
\text { Genebra (Suíça); } \\
\text { Participantes: } 243 \text { médicos, entre } \\
\text { eles } 83 \text { Médicos gerais, } 105 \\
\text { Médicos internistas, } 5 \text { Oncologistas, } \\
\text { 50 Obstetras-ginecologistas. }\end{array}$ & $\begin{array}{l}\text { Conhecimento: Os médicos }(99 \%) \text { afirmaram ter } \\
\text { conhecimento de que é seu papel participar das etapas } \\
\text { pré-teste, divulgação dos resultados, e cuidados e apoio } \\
\text { a longo prazo sobre o teste genético apesar de apenas } \\
32 \% \text { terem respondido o questionário de forma correta. } \\
\text { Atitudes: } 87 \% \text { dos participantes apoiaram o teste } \\
\text { genético. }\end{array}$ \\
\hline A $3^{(20)}$ & $\begin{array}{lll}\text { Descrever } & \text { as } & \text { características } \\
\text { demográficas } & \text { e práticas dos médicos } \\
\text { participantes } & \text { e não participantes do } \\
\text { programa de } & \text { rastreamento, bem como }\end{array}$ & $\begin{array}{l}\text { Tipo de estudo: Transversal, } \\
\text { descritivo quantitativo; } \\
\text { Local de estudo: EUA; } \\
\text { Participantes: } 1.111 \text { médicos, entre }\end{array}$ & $\begin{array}{l}\text { Conhecimento: A maioria dos médicos do programa } \\
\text { acreditava que o exame clínico das mamas e o } \\
\text { autoexame das mamas eram efetivamente eficazes e que } \\
\text { a mamografia (ou mamografia digital) para mulheres }\end{array}$ \\
\hline
\end{tabular}




\begin{tabular}{|c|c|c|c|}
\hline & $\begin{array}{l}\text { suas crenças, adoção de novas } \\
\text { tecnologias de triagem e recomendações } \\
\text { para rastreio do câncer de mama e colo } \\
\text { do útero. }\end{array}$ & $\begin{array}{l}\text { eles, Médicos da família, Médicos } \\
\text { gerais, Médicos internistas, } \\
\text { Obstetras-ginecologistas. }\end{array}$ & $\begin{array}{l}\text { com mais de } 50 \text { anos era muito eficaz. } \\
\text { Atitudes: Os médicos que participam do programa eram } \\
\text { mais propensos em trabalhar com equipe } \\
\text { multiespecialidades. } \\
\text { Práticas: Especificamente para o câncer de mama, o } \\
\text { estudo mostrou que } 61 \% \text { dos médicos do programa e } \\
72 \% \text { dos médicos que não participam do programa } \\
\text { recomendam mamografia anual para mulheres entre } 40 \\
\text { a } 49 \text { anos. }\end{array}$ \\
\hline $\mathrm{A} 4^{(21)}$ & $\begin{array}{l}\text { Examinar a prática e as necessidades } \\
\text { futuras dos médicos sobre a avaliação do } \\
\text { risco de câncer de mama. }\end{array}$ & 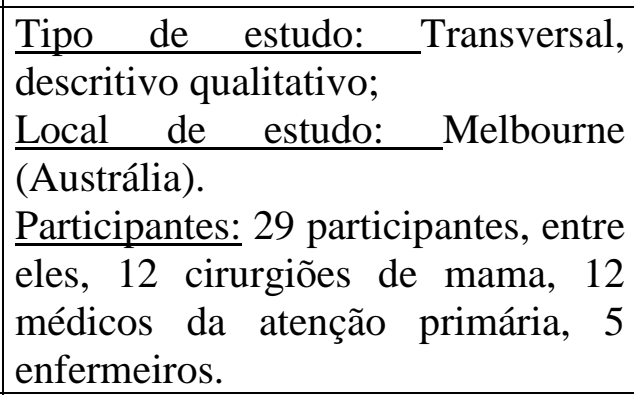 & $\begin{array}{l}\text { Atitudes: Os profissionais preferem não apresentar a } \\
\text { avaliação de risco de câncer pois os possíveis danos são } \\
\text { maiores que os benefícios e ainda não há uma } \\
\text { recomendação oficial para tal prescrição em primeira } \\
\text { instância. }\end{array}$ \\
\hline
\end{tabular}




\begin{tabular}{|c|c|c|c|}
\hline & & & $\begin{array}{l}\text { Práticas: } 75,7 \% \text { dos médicos realizavam práticas de } \\
\text { triagem superior as recomendadas pela Força Tarefa de } \\
\text { Serviços Preventivos dos EUA (mamografia - bienal de } \\
50 \text { a } 74 \text { anos). }\end{array}$ \\
\hline A6 ${ }^{(23)}$ & $\begin{array}{l}\text { Determinar o nível de conhecimento das } \\
\text { trabalhadoras de saúde primária sobre } \\
\text { câncer de mama e revelar suas atitudes e } \\
\text { comportamentos sobre o autoexame e } \\
\text { mamografia das mamas. }\end{array}$ & 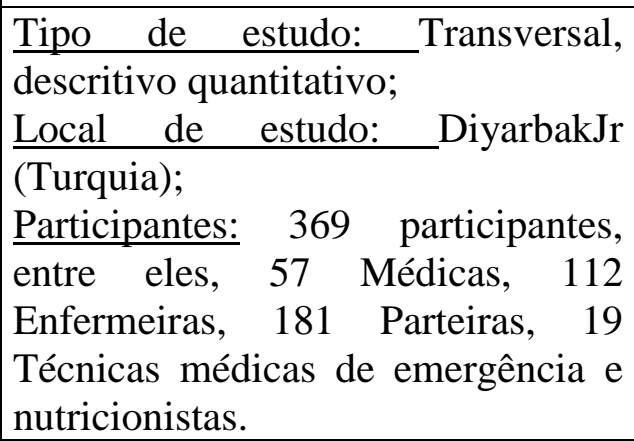 & $\begin{array}{l}\text { Conhecimento: O nível de conhecimento em relação ao } \\
\text { autoexame foi alto, porém sobre o câncer de mama e } \\
\text { mamografia foram baixos. } \\
\text { Atitudes: Das profissionais que realizavam o } \\
\text { autoexame, apenas } 30,1 \% \text { realizavam regularmente. } \\
\text { Práticas: } 92,6 \% \text { das profissionais realizavam o } \\
\text { autoexame das mamas. }\end{array}$ \\
\hline A7 ${ }^{(24)}$ & $\begin{array}{l}\text { Avaliar a triagem mamográfica entre as } \\
\text { trabalhadoras de saúde nos centros de } \\
\text { atenção primária e identificar os } \\
\text { principais motivadores e barreiras que } \\
\text { afetam os resultados de captação das } \\
\text { mulheres. }\end{array}$ & $\begin{array}{l}\text { Tipo de estudo: Transversal, } \\
\text { descritivo quantitativo; } \\
\text { Local de estudo: Cisjordânia; } \\
\text { Participantes: 271 participantes, } \\
\text { entre eles, 15 Médicos, } 229 \\
\text { Enfermeiros, } 8 \text { Farmacêuticos, } 13 \\
\text { Técnicos de laboratório, } 6 \text { não } \\
\text { identificados. }\end{array}$ & $\begin{array}{l}\text { Conhecimento: } 63,4 \% \text { apresentaram conhecimento } \\
\text { sobre a doença e a mamografia excelente, } 31,7 \% \\
\text { conhecimento moderado e } 4,8 \% \text { conhecimento pobre. } \\
\text { Atitudes (em relação a si mesmo): A motivação para } \\
\text { realização da mamografia se baseou no conhecimento } \\
\text { sobre a importância para a sua gestão e que a } \\
\text { mamografia pode detectar câncer de mama antes que } \\
\text { seus sintomas apareçam. Além disso foi verificado se as } \\
\text { profissionais já tinham feito mamografia, onde } 50 \% \text { já } \\
\text { tinham, e destes, 10\% fizeram de forma regular e } 40 \% \\
\text { de forma irregular. As outras 50\% não fizeram por } \\
\text { estarem ocupadas e/ou não acharem que tem câncer de } \\
\text { mama. }\end{array}$ \\
\hline A8 ${ }^{(25)}$ & $\begin{array}{l}\text { Examinar se a crença dos profissionais } \\
\text { de saúde da atenção primária sobre a } \\
\text { eficácia e a frequência de } \\
\text { mamografia de triagem está associada à }\end{array}$ & $\begin{array}{l}\text { Tipo de estudo: Transversal, } \\
\text { descritivo quantitativo; } \\
\text { Local de estudo: Boston, Líbano, } \\
\text { Filadélfia e Vermont (EUA); }\end{array}$ & $\begin{array}{l}\text { Conhecimento: } 50,2 \% \text { dos profissionais acreditavam } \\
\text { que a mamografia para mulheres entre } 49 \text { a } 74 \text { anos era } \\
\text { muito eficaz, } 45,9 \% \text { acreditavam que para a faixa etária } \\
\text { de } 40 \text { a } 48 \text { anos era pouco eficaz e } 30,6 \% \text { para a idade }\end{array}$ \\
\hline
\end{tabular}




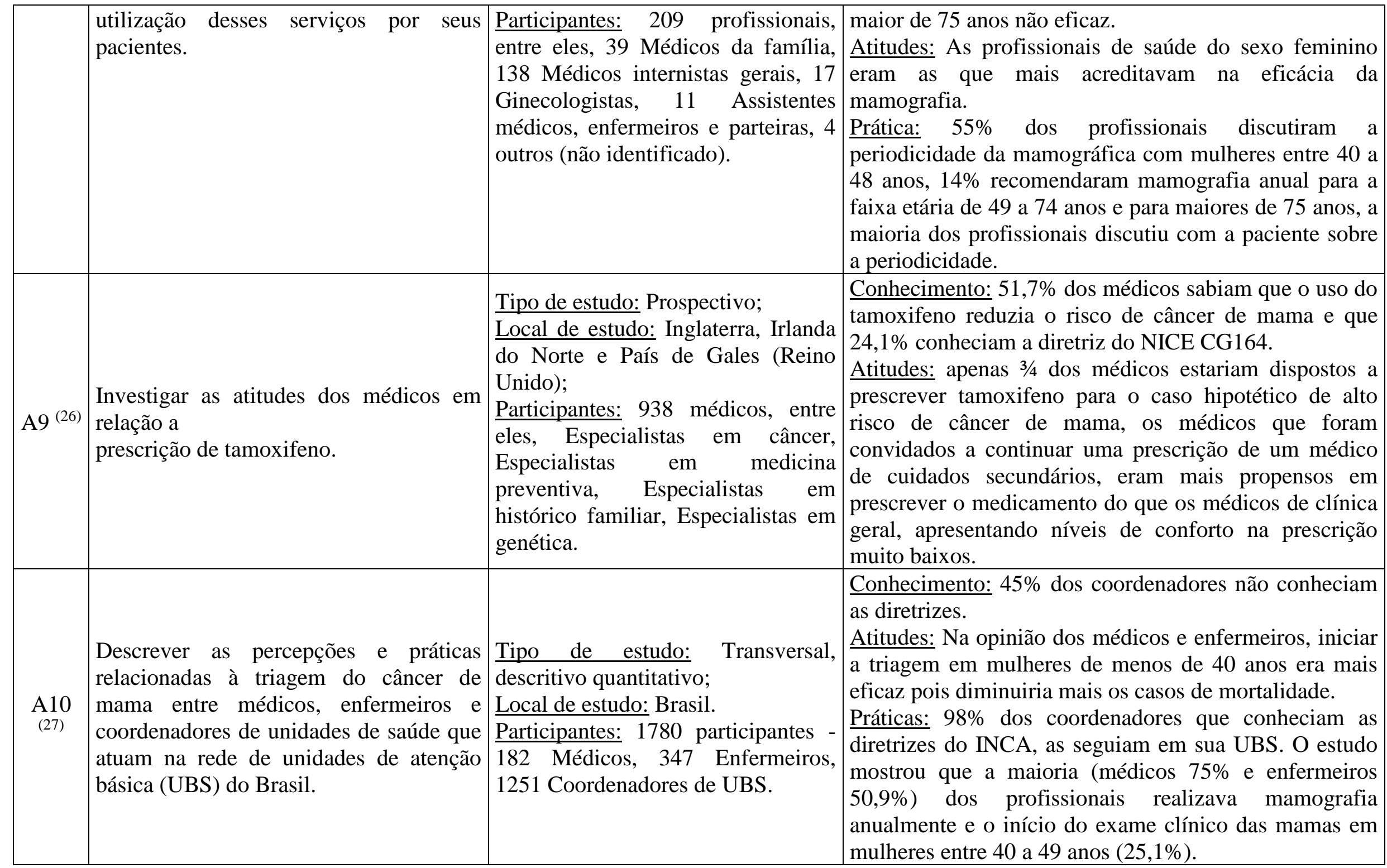




\begin{tabular}{|c|c|c|c|}
\hline$\underset{(28)}{\text { A11 }}$ & $\begin{array}{l}\text { Verificar as perspectivas dos médicos em } \\
\text { relação a realização de exames de rotina } \\
\text { para câncer (mama, colorretal e próstata) } \\
\text { em idosos com expectativa de vida } \\
\text { inferior a } 10 \text { anos. }\end{array}$ & $\begin{array}{l}\text { Tipo de estudo: Transversal, } \\
\text { qualitativo, descritivo; } \\
\text { Local de estudo: Marylan, EUA; } \\
\text { Participantes: } 30 \text { médicos - } 17 \\
\text { médicos internistas, } 6 \text { médicos da } \\
\text { família, 2 médicos pediatras, } 5 \\
\text { médicos geriatras. }\end{array}$ & $\begin{array}{l}\text { Atitudes: Alguns médicos }(60 \%) \text { perceberam o excesso } \\
\text { de realização de exames de rotina em idosos com } \\
\text { expectativa de vida inferior a } 10 \text { anos, como um } \\
\text { problema. Houve polemica quanto a recomendação das } \\
\text { diretrizes de não fazer os exames para esta população, } \\
\text { pois os médicos acreditam que se pode sim diminuir a } \\
\text { mortalidade por câncer nessa faixa etária, além disso } \\
\text { não leva em conta a vontade do paciente e ainda que as } \\
\text { previsões de expectativa de vida são imprecisas. } \\
\text { Acreditam também que utilizar a expectativa de vida } \\
\text { como referência para não realizar os exames de } \\
\text { rastreamento de rotina, pode causar viés e danos não } \\
\text { intencionais. }\end{array}$ \\
\hline
\end{tabular}

Fonte: Elaborado pelos autores. 


\section{DISCUSSÃO}

As ações de controle do câncer de mama vêm sendo progressivamente incorporadas às políticas públicas desde o final dos anos 1980, como uma das diretrizes da atenção integral à saúde da mulher ${ }^{(29)}$. Nesse cenário, de acordo com os autores ${ }^{(30)}$, a Atenção Primária à Saúde (APS) se destaca pelo seu importante papel de promoção do cuidado centrado no desenvolvimento do vínculo, longitudinalidade, coordenação e integralidade da atenção, sendo fundamental na assistência.

Nesse contexto, uma das ferramentas mais utilizadas nesse nível de atenção são, conforme os autores ${ }^{(31)}$, as ações de educação em saúde, que tem como papel primordial transmitir o conhecimento para a população, neste caso em específico sobre o câncer de mama, com o intuito de incentivar comportamentos preventivos, sendo substanciais para as mudanças dos hábitos de vida. Entretanto, as dificuldades em efetivar essas ações, como a falha na atitude e prática profissional, contribuem significativamente para o aumento da incidência e da mortalidade pela neoplasia, o que demonstra ainda a fragilidade desse nível de atenção à saúde ${ }^{(32)}$.

Apesar dos avanços relacionados à prevenção primária do câncer de mama, alguns estudos ${ }^{(20,21,26)}$, pontuam que muitos profissionais de saúde não se sentem capacitados para colocar em prática as ações de educação em saúde para a população, sendo a segurança da atitude essencial nesse processo, pois relataram dificuldades em a partir desse perspectiva avaliar as variáveis relacionadas ao risco do desenvolvimento da neoplasia em questão, bem como orientar àquelas com grau de susceptibilidade elevado para o surgimento da doença. Neste sentido, de acordo o autor ${ }^{(33)}$, é imprescindível a realização da capacitação dos profissionais para que estes possam lidar com a neoplasia mamária no atendimento, desenvolvendo as atitudes adequadas, pois investimentos em ações de prevenção, bem como detecção precoce e implementação de programas efetivos no controle da doença são condutas fundamentais.

Os programas de controle do câncer de mama estão centrados principalmente em dois componentes, e de extrema importância os profissionais conhecerem: o diagnóstico precoce e o rastreamento. $\mathrm{O}$ diagnóstico precoce relaciona-se às ações que identificam indivíduos sintomáticos em estágios iniciais, já o rastreamento diz respeito às atitudes voltadas aos indivíduos assintomáticos, objetivando detectar a doença e/ou lesões precursoras em fase inicial (34). O rastreamento é oferecido de diferentes formas em diversos países, desde o oportunístico que é realizado por ocasião de uma consulta ou 
planejado através da busca ativa da população alvo ${ }^{(35)}$.

De acordo com as Diretrizes de Detecção Precoce do Câncer de Mama no Brasil (34), o rastreamento mamográfico na clínica é um exame utilizado para avaliar se há indícios de lesões mamárias em mulheres na faixa etária entre 50 a 69 anos, a cada dois anos, pois fora dessa periodicidade e da faixa etária estabelecida, os riscos são maiores e os benefícios são incertos. Em contrapartida, a Sociedade Brasileira de Mastologia (37), recomenda que a mamografia pode ser realizada em mulheres assintomáticas de 40 até 75 anos de idade, com periodicidade anual ou bienal, variando em diferentes países. Um outro método que é preconizado pelo Ministério da Saúde no atendimento à mulher é o exame clínico das mamas, que consiste na inspeção e a palpação pelo profissional, visando detectar anormalidades evidentes principalmente em fases iniciais ${ }^{(38)}$.

Diante dessas diferentes recomendações pelos órgãos respaldados, pode ser apontada como uma barreira para o rastreamento e encaminhamento para realização da mamografia, o conhecimento dos profissionais á cerca de uma recomendação única e unanime, assim como verificado no estudo (18), pois para mamografia segundo o Instituto Nacional do Câncer deve ser anualmente para mulheres com 50 anos ou mais e a Força Tarefa de
Serviços Preventivos dos EUA, preconizava que nesta faixa etária em mulheres com baixo risco, o intervalo poderia ser bienal, nessa mesma época. Percebe-se que essa dificuldade que anseia sobre os profissionais relacionada à recomendações diferentes, já perpassa ao longo das décadas.

Já no estudo ${ }^{(25)}$, afirmou-se que, a maioria dos profissionais passaram a discutiram com a paciente sobre a periodicidade da realização da mamografia, mesmo com a existência dessa barreira. Portanto, esses profissionais precisam conhecer as recomendações, para assim subsidiar apoio para as pacientes, na qual possam ter o domínio sobre os procedimentos de triagem e prevenção, podendo promover uma diminuição das barreiras existente relacionada ao rastreamento e atendimento á mulheres.

De modo geral, sete estudos apresentaram dados sobre o conhecimento dos profissionais de saúde em relação as ações de rastreamento e detecção precoce do câncer de mama, onde cinco apresentaram conhecimento adequado pela maioria dos participantes (18,20,24-25,27), e apenas dois conhecimento inadequado (22-23). Quanto as atitudes, foram apresentadas em oito estudos, sendo em dois considerado corretas ${ }^{(18,26)}$ e em seis incorretas (22-25,27-28). Em relação as práticas dos profissionais de saúde, seis estudos trouxeram dados, demonstrando que 
em apenas um apresentou prática correta pela maioria dos profissionais (23) e em cinco estudos incorretas (18,20,22,25,27). O que demonstra que apesar de os profissionais terem apresentado conhecimento adequado na maioria dos estudos, isso não é refletido em suas atitudes e práticas, visto que a maioria apresentou essas variáveis incorretas.

Esses conhecimentos, atitudes e práticas, estão relacionados, em sua maioria dentro dos estudos, com a doença, e as recomendações de exames, onde principalmente a idade e periodicidade da mamografia foram bastante discutidas, sendo que a realização adequada de acordo com o INCA, é bienal em mulheres de 50 a 69 anos, pois dessa forma não sobrecarrega o sistema de triagem, aumenta a cobertura do programa de triagem para as mulheres, e assim, diminui o percentual de mortalidade da doença ${ }^{(34)}$.

Apenas os estudos dos autores $(26,19,21)$ apresentaram contextos diferentes, sobre prescrição de tamoxifeno, testes genéticos e avaliação de risco de câncer, respectivamente. Sendo que no estudo (21), a maioria dos participantes disseram preferir não apresentar a avaliação de risco das pacientes, o que é considerado uma atitude incorreta, pois os indivíduos tem direto de ser informados sobre tudo, e a partir disso, tomarem uma decisão informada sobre suas práticas de triagem ${ }^{(34,36)}$. No estudo ${ }^{(26)}$, a maioria dos médicos sabiam que o tamoxifeno reduzia o risco de desenvolver câncer de mama, considerado um conhecimento adequado e que a maioria estavam dispostos a prescrever $\mathrm{O}$ quimiopreventivo, atitude correta, de acordo com a recomendação da SBM (36). Já a pesquisa ${ }^{(19)}$, abordaram sobre testes genéticos para a doença, onde apenas $32 \%$ dos participantes responderam corretamente aos conhecimentos sobre o assunto e $87 \%$ disseram apoiar esses testes, recomendados pela SBM para casos específicos ${ }^{(36)}$.

Os estudos dos autores (23,24), revelaram que as atitudes e práticas dos profissionais consigo são insuficientes, onde nem todos os profissionais (mulheres) realizam os exames necessários, e as que realizam, os fazem de forma irregular. Ressalta-se a importância de o profissional de saúde ser um modelo ao paciente, realizando o recomendado de forma eficiente e respaldada para seu autocuidado.

Percebe-se que, embora as diretrizes desenvolvidas por cada país sejam respaldadas por estudos, normas levantadas e discutidas através de consensos sobre as recomendações, baseadas em evidências, diversos profissionais ainda as ignoram, em detrimento de suas opiniões e práticas adquiridas na rotina, por determinar ainda, que não é necessário a realização da triagem preventivas, devido a idade da paciente, discordando em percepção e prática das diretrizes ${ }^{(22,26,28)}$. Isso revela uma postura 
fragilizada, afetando a atitude dos profissionais quanto ao seu papel na detecção precoce do câncer de mama.

O estudo ${ }^{(25)}$ trouxe um levantamento importante, onde foi verificado que as profissionais de saúde do sexo feminino foram as que mais acreditavam na eficácia da mamografia. Sendo assim, conscientizar profissionais homens, através da troca do dialogo educacional, é um importante elemento para aumentar eficácia dos programas preventivos, para promover boa prática no atendimento á mulheres.

Além dos profissionais que atuam diretamente no atendimento, na realização de consultas, é essencial que toda a equipe tenha um mínimo de conhecimento sobre o câncer de mama e sua prevenção, como os coordenadores, e outros profissionais, conseguindo esclarecer, encaminhar ou solicitar atendimento ${ }^{(18,20)}$. Neste sentido, as ferramentas educativas ou de assistência, podem ajudar a remover barreiras de insegurança nas equipes de saúde, e aumentar a convicção para uma boa atitude e melhor preparo profissional na atenção em como falar com as mulheres e as possibilidades de práticas preventivas ${ }^{(21)}$.

Posto isso, ressalta-se a importância da capacitação profissional para que estes saibam identificar com veracidade as características anatômicas da mama e suas possíveis alterações quando há indícios sugestivos de uma neoplasia, se sentirem aptos e seguros para a realização dos exames de prevenção do câncer de mama, e basearem suas atitudes e práticas no conhecimento adequado sobre a doença e as ações de prevenção. Além disso, é fundamental a proatividade destes profissionais no planejamento e implementação das ações de educação em saúde para buscar o empoderamento dos indivíduos e adquirir autonomia profissional para promover o controle da doença.

\section{CONSIDERAÇÕES FINAIS}

O conhecimento analisado na maioria dos estudos foi considerado adequado, porém isso não foi refletido nas atitudes e práticas. Sendo que, fatores como discordância das diretrizes, conhecimento fragmentando e sentimento de insegurança para o aconselhamento ou realização dos exames, se apresentaram como interferentes na tomada de decisões dos profissionais. Diante desse contexto, é importante reforçar a ligação da atitude e prática com o conhecimento adquirido, na qual se tornam um tripé para a eficácia da abordagem junto a mulher para a prevenção do câncer.

Cabe ressaltar que o conhecimento, as atitudes e as práticas dos profissionais de saúde da atenção primária sobre prevenção do câncer de mama devem estar alinhados e 
sobretudo em concordância com as diretrizes e normas estabelecidas através dos estudos e discursões cientificas levantadas. Além disso, é necessário que os profissionais estejam em contínua atualização, abertos para diálogos e novas percepções.

\section{AGRADECIMENTOS}

Á Coordenação de Aperfeiçoamento de Pessoal de Nível Superior -Brasil (CAPES) - Código de Financiamento 001. À Fundação de Amparo à Pesquisa e ao Desenvolvimento Científico e Tecnológico do Maranhão (FAPEMA) pelo financiamento da pesquisa e das bolsas de Iniciação Científica e Mestrado.

\section{REFERÊNCIAS}

1 Campos SAPBM, Almeida CAPL, Lago EC, Tapety FIZ, Alves ELM. Percepções de médicos sobre a prevenção do câncer de mama. Rev de Enfermagem UFPE Online [Internet]. 2019; [acesso em 21 jun 2020]; 13(2). Disponível em: https://periodicos.ufpe.br/revistas/revistae nfermagem/article/viewFile/235056/3127 4

2 Ferreira DDS, Bernardo FMDS, Costa EC, Maciel NDS, Costa RLD, Carvalho CMDL. Conhecimento, atitude e prática de enfermeiros na detecção do câncer de mama. Rev Escola Anna Nery [Internet]. 2020; [acesso em 21 jun 2020]; 24(2). Disponível em: https://www.scielo.br/j/ean/a/fcH45Y8Q8 HPfLqWFKKCmbMr/?lang=pt

3 Oliveira MLC, Gomes LO, Silva, HS, Chariglione, IPFS. Conhecimento, atitude e prática: conceitos e desafios na área de educação e saúde. Rev Educação em saúde [Internet]. 2020; [acesso em 10 ago 2020]; 8(1):190-198. Disponível em: https://core.ac.uk/download/pdf/32714530 3.pdf

4 Ferlay J, Colombet M, Soerjomataram I, Mathers C, Parkin DM, Piñeros M., Bray F. Estimating the global cancer incidence and mortality in 2018: GLOBOCAN sources and methods. Int. J. Cancer [Internet]. 2019; [acesso em 10 ago 2020]; (144):1941-1953. Disponível em: https://onlinelibrary.wiley.com/doi/abs/10. 1002/ijc.31937

5 Binotto M, Schwartsmann G. Qualidade de Vida Relacionada à Saúde de Pacientes com Câncer de Mama: Revisão Integrativa da Literatura. Rev Brasileira de Cancerologia [Internet]. 2020; [acesso em dez 2020]; 66 (1). Disponível em: https://rbc.inca.gov.br/revista/index.php/re vista/article/view/405

6 Munhoz MP, Oliveira JD, Gonçalves R D, Zambon TB, Oliveira LCND. Efeito do exercício físico e da nutrição na prevenção do câncer. Rev Odontológica de Araçatuba [Internet]. 2016; [acesso em 10 jan 2021]. 37(2):09-16. Disponível em: https://apcdaracatuba.com.br/revista/2016/ 08/trabalho5.pdf

7 Melillo CD, Morais PLMÁ, Aleixo TB, Machado RRP, Figueiredo ACDS. Perfil epidemiológico das pacientes com câncer de mama atendidas em Juiz de ForaMinas Gerais (MG), Brasil. Brazilian Journal of Development [Internet]. 2020; [acesso em 10 jan 2021]. 6(10):8057580592. Disponível em: https://www.brazilianjournals.com/index. php/B RJD/article/view/18626/14998.

8 Loyola EAC, Panobianco MS. Vigilância à Saúde: práticas no controle do câncer de mama identificadas por gestores da Atenção Primária à Saúde 
[Tese doutorado]. [São Paulo]: Universidade de São Paulo- Ribeirão Preto; 2017. Disponível em: http://www.teses.usp.br/teses/disponiveis/ 22/22133/tde-28032018-154426.

9 Melo FBB, Figueiredo END, Panobianco MS, Gutiérrez MGRD, Rosa ADS. Detección precoz de cáncer de mama en unidades básicas de salud. Rev Acta paul. Enfer [Internet]. 2021; [acesso em 10 mai 2021]; (34):1982-0194. Disponível em: https://www.scielo.br/j/ape/a/b85WmQY K4dbmLFPnT3TwnVs/?format=html\&lan $\mathrm{g}=\mathrm{pt}$

10 Farias JM. Minghelli LC, Soratto J. Promoção da saúde: discursos e concepções na atenção primária à saúde. Cadernos saúde coletiva [Internet]. 2020; [acesso em 10 mai 2021]; 28(8):381-389. Disponível em: https://www.scielo.br/j/cadsc/a/VPxk9Pg X9xQxHqCLDZqwFhF/?lang=pt

11 Heena H, Durrani S, Riaz M, AlFayyad I, Tabasim R, Parvez G, AbuShaheen A. Knowledge, attitudes, and practices related to breast cancer screening among female health care professionals: a cross sectional study. BMC women's health [Internet]. 2019; [acesso em 10 mai 2021]; 19(1):1-11. Disponível em: https://bmcwomenshealth.biomedcentral.c om/articles/10.1186/s12905-019-0819-x.

12 Mekonnen BD. Breast selfexamination practice and associated factors among female healthcare workers in Ethiopia: A systematic review and meta-analysis. PLoS One [Internet]. 2020; [acesso em 15 mai 2021]; 15 (11). Disponível em: https://doi.org/10.1371/journal.pone.0241 961.

13 Jorgensen L, Kastrup JS, Brogaard B. Situational awareness in the outpatient encounter between patients with breast cancer or malignant melanoma and healthcare professionals: Patients' perceptions. Journal of clinical nursing [Internet]. 2020; [acesso em 15 mai 2021]; 29(11):1981-1990. Disponível em: https://onlinelibrary.wiley.com/doi/abs/10. 1111/jocn. 15122

14 Marchito LO, Fabro EAN, Macedo FO, Costa RM, Lou MBA. Prevenção e Cuidado do Linfedema após Câncer de Mama: Entendimento e Adesão às Orientações Fisioterapêuticas. Rev Brasileira de Cancerologia [Internet]. 2019; [acesso em 15 mai 2021]; 65(1). Disponível em: https://rbc.inca.gov.br/revista/index.php/re vista/article/view/273

15 Sousa LMM, Marques-Vieira CMA, Severino SSP, Antunes AV. Metodologia de revisão integrativa da literatura em enfermagem. Rev investigação em enfermagem [Internet]. 2017; [acesso em 10 dez 2020]; 1311(1). Disponível em: http://www.sinaisvitais.pt/images/stories/ Rie/RIE21.pdf\#page $=17$

16 Joanna BI. (JBI). Joanna Briggs Institute reviewers' manual: 2019 edition. The Joanna Briggs Institute. UK. 2019. Disponível em: https://wiki.joannabriggs.org/display/MA NUAL/2.6.2+Review+question

17 Galvão TF, Pansani TSA, Harrad D. Principais itens para relatar Revisões sistemáticas e Meta-análises: A recomendação PRISMA. Rev Epidemiologia e Serviços de Saúde [Internet]. 2015; [acesso em $10 \mathrm{dez} 2020$ ]; 24(2):335-342. Disponível em: https://www.scielosp.org/article/ssm/conte nt/raw/?resource_ssm_path=/media/assets /ress/v24n2/2237-9622-ress-24-02-

00335.pdf

18 Taylor VM, Taplin SH, Urban N, Mahloch J, Majer KA. Medical 
Community Involvement in a Breast Cancer Screening Promotional Project. Public Health Reports [Internet]. 1994; [acesso em 12 jan 2021]; 109(4):491-499. Disponível em: https://www.ncbi.nlm.nih.gov/pmc/article s/PMC1403525/

19 Escher M, Sappino AP. Primary care physicians' knowledge and attitudes towards genetic testing for breast-ovarian cancer predisposition. Kluwer Academic Publisher Annals of Oncology [Internet]. 2000; [acesso em 12 jan 2021]; 11(9):1131-1135. Disponivel em: https://doi.org/10.1023/A:1008319114278

20 Benard VB, Saraiya MS, Soman A, Roland KB, Yabroff KR, Miller J. Cancer Screening Practices Among Physicians in the National Breast and Cervical Cancer Early Detection Program. Journal of Women's Health [Internet]. 2001; [acesso em 13 jan 2021]; 20(10):1479-1484. Disponível em: https://www.liebertpub.com/doi/abs/10.10 89/jwh.2010.2530

21 Collins IM, Steel E, Mann GB, Emery JD, Bickerstaffe A, Trainer A. Assessing and managing breast cancer risk: Clinicians' current practice and future needs. The Breast [Internet]. 2014; [acesso em 13 jan 2021] 23:644-650 http://dx.doi.org/10.1016/j.breast.2014.06. 014

22 Haas JS, Sprague BL, Klabunde CN, Tosteson ANA, Chen JS, Bitton A. Provider Attitudes and Screening Practices Following Changes in Breast and Cervical Cancer Screening Guidelines. Journal of General Internal Medicine [Internet]. 2015; [acesso em 13 jan 2021]; 31(1)52-59. Disponível em: https://link.springer.com/content/pdf/10.1 007/s11606-015-3449-5.pdf
23 Erdem O, Toktas E. Knowledge, Attitudes, and Behaviors about Breast Self-Examination and Mammography among Female Primary Healthcare Workers in DiyarbakJr, Turkey. BioMed Research International [Internet]. 2016; [acesso em 20 jan 2021]. Disponível em: https://www.hindawi.com/journals/b mri/2016/6490156/

24 Nazzal Z, Sholi H, Sholi S, Sholi M, Lahaseh R. Mammography Screening Uptake among Female Health Care Workers in Primary Health Care Centers in Palestine - Motivators and Barriers. Asian Pacific Journal of Cancer Prevention [Internet]. 2016; [acesso em 20 jan 2021]; 17. Disponível em: http://journal.waocp.org/article_32436.ht $\mathrm{ml}$

25 Haas JS, Barlow WE, Schapira MM, Maclean CD, Klabunde CN, Sprague BL. Primary Care Providers' Beliefs and Recommendations and Use of Screening Mammography by their Patients. Journal of General Internal Medicine [Internet]. 2017; [acesso em 20 jan 2021]; 32(4):449-457. Disponível: https://link.springer.com/content/pdf/10.1 007/s11606-016-3973-y.pdf

26 Smith SG, Foy R, Mcgowan JA, Kobayashi LC, Decensi A, Brown K. Prescribing tamoxifen in primary care for the prevention of breast cancer: a national online survey of GPs' attitudes. British Journal of General Practice [Internet]. 2017; [acesso em 22 jan 2021]. Disponível em: https://bjgp.org/content/67/659/e414.short

27 Hallowella BD, Perina DMP, Simoesb EJ, Paezc DC, Parrac DC, Brownson RC. Breast cancer related perceptions and practices of health professionals working in Brazil's network of primary care units. Preventive Medicine [Internet]. 2018; [acesso em 25 jan 2021]; 106:216-223. Disponível

em: 
https://www.sciencedirect.com/science/art icle/pii/S0091743517304243

28 Schoenborn NL, Massare J, Park R, Pollack CE, Choi Y, Boyd M. Clinician Perspectives on Overscreening for Cancer in Older Adults With Limited Life Expectancy. The American Geriatrics Society JAGS [Internet]. 2020; [acesso em 25 jan 2021]; 68(7) 2020. Disponível em:

https://agsjournals.onlinelibrary.wiley.co m/doi/abs/10.1111/jgs.16415

29 Tomazelli JG, Migowski A, Ribeiro CM, Assis M, Abreu DMF. Avaliação das ações de detecção precoce do câncer de mama no Brasil por meio de indicadores de processo: estudo. Rev Epidemiol. Serv. Saúde [Internet]. 2017; [acesso em $02 \mathrm{fev}$ 2021]; 26(1). Disponível em: https://doi.org/10.5123/s1679-

49742017000100007

30 Souza GRM, Cazola LHO, Pícoli RP. Atuação do Enfermeiro na Atenção Primária à Saúde na Assistência Oncológica: Revisão Integrativa. Rev Cogitare Enfermagem [Internet]. 2018; [acesso em 02 fev 2021]; 23(4). Disponível em: https://www.redalyc.org/jatsRepo/4836/48 3660655024/483660655024.pdf

31 Silva ARS, Alves RP, Barros EBSC, Bushatsky M, Souto MMR, Filho CMSF, Simão A. Educação em saúde para detecção precoce do câncer de mama. Rev Rene [Internet]. 2011; [acesso em $02 \mathrm{fev}$ 2021]; 12:952-959. Disponível em: https://www.redalyc.org/articulo.oa? $\mathrm{id}=32$ 4027978009

32 Schlemmer JB, Castilhos LG, Lima SBS. Políticas Públicas e a Atuação dos Gestores Frente ao Câncer de Mama e do Colo Uterino. Rev Saúde (Santa Maria) [Internet]. 2016; [acesso em 09 fev 2021]; p.53-62.

Disponível https://www.researchgate.net/publication/ 305484739

33 Moraes DC, Almeida AM, Figueiredo EN, Loyola EAC, Panobianco MS. Opportunistic screening actions for breast cancer performed by nurses working in primary health care. Rev Esc Enferm USP [Internet]. 2019; [acesso em $10 \mathrm{fev} 2021$ ]; 50(1):14-21. Disponível em: https://www.scielo.br/j/reeusp/a/BTJXVq HgTcbqCGfSXTxNNCC/?lang=en\&form at=html

34 Instituto Nacional De Câncer José Alencar Gomes Da Silva (BR). Diretrizes para a detecção precoce do câncer de mama no Brasil. Rio de Janeiro, INCA. 2015; [acesso em 22 dez 2020]. Disponível em: https://www.inca.gov.br/controle-docancer-de-mama/acoes-decontrole/deteccao-precoce

35 Silva RCF, Hortale VA. Rastreamento do Câncer de Mama no Brasil: Quem, Como e Por quê? Rev Brasileira de Cancerologia [Internet]. 2021; [acesso em 12 fev 2021]; 58(1):67-71. Disponível em: http://www1.inca.gov.br/rbc/n_58/v01/pdf /10b_artigo_opiniao_rastreamento_cancer _mama_brasil_quem_como_por_que.pdf

36 Sociedade Brasileira De Mastologia (BR). Câncer de Mama: Consenso da Sociedade Brasileira de Mastologia: Regional Piauí, SBM. 2017; [acesso em 12 fev 2021]. Disponível em: https://www.sbmastologia.com.br/medico s/wpcontent/uploads/2018/03/C\%C3\%A2ncerde-Mama-Consenso-da-SBM-RegionalPiau\%C3\%AD-2017.pdf

37 Sociedade Brasileira De Mastologia (BR). Rastreamento Mamográfico. SBM. 2019; [acesso em 12 fev 2021]. Disponível em: https://www.sbmastologia.com.br/noticias 
/nota-oficial-informacoes-distorcidas-damamografia-nas-redes-sociais/

38 Ministério da Saúde (BR). Protocolos da Atenção Básica: Saúde das Mulheres/ Instituto Sírio-Libanês de Ensino e Pesquisa. Ministério da Saúde, Brasília, DF. 2016; [acesso em 13 fev 2021]. Disponível em: http://bvsms.saude.gov.br/bvs/publicacoes /protocolos_atencao_basica_saude_mulhe res.pdf

\section{"Autor correspondente}

Aldo Lopes da Costa Júnior. Endereço: Rua Piauí 449 CEP: 65907100, Imperatriz, Maranhão. Cel.: (99) 98234-5315.

Submissão: 2021-09-01

Aprovado: 2021-08-10 